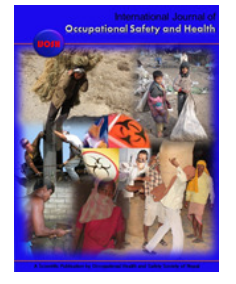

Available Online at http://nepjol.info/index.php/IJOSH

Editorial

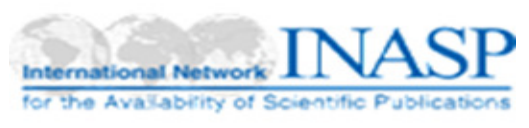

International Journal of Occupational Safety and Health, Vol 1 (2011) 1 - 2

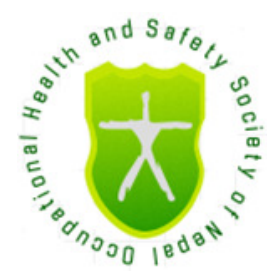

\title{
Occupational Safety and Health in Nepal
}

\author{
Dr. Sunil Kumar Joshi Grad IOSH \\ Chief Editor, International Journal on Occupational Safety and Health (IJOSH)
}

Since 1950, the International Labor Organization (ILO) and the World Health Organization (WHO) have shared a common definition of occupational health. This definition was adopted by the Joint ILO/WHO Committee on Occupational Health at its first session in 1950 and revised at its twelfth session in 1995. The definition reads: "Occupational health should aim at: the promotion and maintenance of the highest degree of physical, mental and social well-being of workers in all occupations; the prevention amongst workers of departures from health caused by their working conditions; the protection of workers in their employment from risks resulting from factors adverse to health; the placing and maintenance of the worker in an occupational environment adapted to his physiological and psychological capabilities; and, to summarize, the adaptation of work to man and of each man to his job."

Occupational Safety and Health (OSH) is a cross-disciplinary area concerned with protecting the safety, health and welfare of people engaged in work or employment. The goal of all occupational safety and health programs is directed in fostering a safe work environment.

$\mathrm{OSH}$ determines vulnerabilities and provides mitigating and adoptive measures to overcome any work related adversities. It analyses problems through surveillance in determining hazards, conditions of work, and exposure of workers. The tools of $\mathrm{OSH}$ assess the health of workers and takes measures to reduce vulnerability of hazards and risk which can cause health impairment. It also dictates on preventive and control measures to prevent unnecessary exposure during normal operating conditions. It also includes emergency preparedness and continues on in case of possible accidents and emergencies. OSH strengthens health education, health promotion, and promotion of work ability through disseminating Information on identified workplace health hazards.

\section{Stakeholders in Occupational Safety and Health}

The concept of OSH in Nepal is in its initial stage. The notion of $\mathrm{OSH}$ in Nepal has not been able to educate about its principle and ideologies at all level. The OSH sector in Nepal has three major identified sectors, 1) government, 2) the industries/ employers and 3) the work force/labor unions.

The government of Nepal has enforced concepts of OSH through its Labor Act 1992; it has highlighted few issues and provisions on working hours, physical infrastructural setup, yearly medical examination and provisions of safety measures in work etc. It has already endorsed 9 conventions passed by ILO but has not yet ratified convention No. 155 which solely bears OSH obligations. In this light, except for few enactments under Labor Act 1992, the issues of OSH still lack legal backup.

The employer or the industries bearing obligation to providing safe working conditions by practicing $\mathrm{OSH}$ have an easy exit mechanism in absence of concrete laws. Few industries have taken prudent measures by establishing OSH setups thereby decreasing vulnerability of hazards. The reality has a different story to tell, with very few medical facilities and emergency medical backup the issues of OSH are largely overlooked.

The labor forces are guided by their political affiliations, lack effective coordination and unity on OSH issues. They have not been able to cater to the needs of much aspired OSH concerns of workers. The labor forces at the bottom rung of ladder are still facing hardship as their voices often fall on deaf ears and issues pertaining to healthy working conditions are overlooked by their employers and even the state. The reality of this situation is such that workers have still been collectively bargaining for issues relating to minimum wages, equal remuneration etc. thereby, overshadowing issues pertaining to occupational safety and health. 


\section{Current Scenario}

The major façade to installing concept of OSH in Nepal lies in inability of concerned stakeholders to grasp the utility and importance of occupational health services. The major impediments in enforcing effective OSH practices in Nepal are as follows:

- $\quad$ Lack of legal back up mechanisms

- $\quad$ Need of separate laws on OSH

- $\quad$ Update of Labor Act 1992

- Isolation and overlooking of OSH issues by formal and informal sectors

- Necessity to build the capacity of State, employers and workers on $\mathrm{OSH}$

\section{Needs}

Any practice needs a valid and justifiable legal support for its effective enforcement and implementation. Nepal has yet to ratify various international declarations and protocols under OSH chiefly ILO convention 155. Absence of firm legal grounds, acts and policies have always produced mayhem, so is the case of $\mathrm{OSH}$ in Nepal. There is an immediate need for enacting OSH specific legal tools. The other strategic needs are as follows:

- Effective legal tools and their enforcements

- Formation of apex government body specifically for $\mathrm{OSH}$

- Dialogue, interactions and brainstorming sessions for policy formulation and necessary amendments

- $\quad$ Training, capacity building and skill transfer

- Detailed studies and publications on OSH in Nepal

\section{Why IJOSH - the first Journal of Occupational Safety and Health in Nepal?}

The task of maintaining a safe and healthy work environment is not an easy alley to trespass. All the stakeholders should be informed and educated on occupational grounds for an effective occupational response through their meaningful participation. The government should develop evidence based legislations, policy, framework, and programs on occupational safety and health. It requires the translation of scientific findings on occupational safety and health into policies. There are few studies being conducted in Nepal on OSH. Most of them go unpublished due to lack of separate journal on OSH in Nepal or rejection from the international journals because of language difficulties, though they contain excellent informative results. Journals too require proficient editors to carry this out. The International Journal of Occupational Safety and Health (IJOSH), the first journal on $\mathrm{OSH}$ in Nepal will be a firm platform containing new information on occupational safety and health for the scientific papers from Nepal and other developing countries. IJOSH will provide doctors, occupational health physicians, ergonomists, nurses, occupational hygienists, health and safety professionals, environmentalists, sociologists and the policy makers with a good source of information on occupational safety and health from Nepal and other countries in the region.

IJOSH welcomes original research submissions which relate to the full range of chemical, physical, ergonomic, biological and psychosocial hazards in the workplace and to environmental contaminants and their health effects and the safety standards and interventions. Research aimed at improving the evidencebased practice of occupational medicine and research on the development and application of novel methods, including biological and statistical techniques are also of interest. In addition, we publish systematic reviews, editorials, commentaries and letters to the editor, which provide a good forum for debate. 